\title{
Signal Amplification by Sensitive Control of Bifurcation Topology
}

\author{
R. B. Karabalin, ${ }^{1}$ Ron Lifshitz, ${ }^{2, *}$ M. C. Cross, ${ }^{1}$ M. H. Matheny, ${ }^{1}$ S. C. Masmanidis, ${ }^{1}$ and M. L. Roukes ${ }^{1, \dagger}$ \\ ${ }^{1}$ Kavli Nanoscience Institute and Condensed Matter Physics, California Institute of Technology, \\ MC 149-33, Pasadena, California 91125, USA \\ ${ }^{2}$ Raymond and Beverly Sackler School of Physics and Astronomy, Tel Aviv University, 69978 Tel Aviv, Israel
} (Received 25 August 2010; published 28 February 2011)

\begin{abstract}
We describe a novel amplification scheme based on inducing dynamical changes to the topology of a bifurcation diagram of a simple nonlinear dynamical system. We have implemented a first bifurcationtopology amplifier using a coupled pair of parametrically driven high-frequency nanoelectromechanical systems resonators, demonstrating robust small-signal amplification. The principles that underlie bifurcation-topology amplification are simple and generic, suggesting its applicability to a wide variety of physical, chemical, and biological systems.
\end{abstract}

DOI: 10.1103/PhysRevLett.106.094102

PACS numbers: 05.45. $-\mathrm{a}, 62.25 .-\mathrm{g}, 85.85 .+\mathrm{j}$

Interest in parametric excitation-whereby resonators are set in motion by dynamically modulating their physical parameters - has grown over the last decade given its many uses. These include parametric amplification, and squeezing of classical and quantum noise [1], generation of entangled pairs of photons [2], and effective actuation of micro- and nanomechanical resonators [3-5]. Our previous investigations of the parametric excitation of coupled nanomechanical resonators $[6,7]$ have led to the discovery of a novel and generic amplification mechanism. Unlike previous schemes employing a static bifurcation [8], this approach is based on inducing dynamical changes to the topology of a simple bifurcation diagram through the application of a small control signal. Thus, rather than positioning the device's oscillation state near a static bifurcation-at a point where abrupt jumps may occur in response to small changes in the input-the control signal induces dynamical changes in the nature of the bifurcation itself, i.e., it changes the way in which different branches of stable solutions are connected to each other. The input signal does not merely cause the system to go through a bifurcation point, thus inducing abrupt jumps, but rather it controls whether a high-response or a low-response stable solution is connected to the initial branch where the device is biased. As explained below, the response thus follows, continuously, either the high or the low branch, amplifying the input signal without any abrupt jumps between states. Here we describe a first implementation of a bifurcation-topology amplifier (BTA) based on a coupled pair of nonlinear high-frequency nanomechanical resonators, actuated piezoelectrically [9] and measured by laser interferometry at room temperature [10].

We consider a single resonator-in our case a nanomechanical doubly-clamped GaAs beam, vibrating in its fundamental transverse mode-and assume that it undergoes weak damping and possesses some nonlinearity both in its potential energy and in its damping. The resonator is driven parametrically by modulating its effective stiffness, the equation of motion is then given by [6]

$$
\ddot{x}+\gamma \dot{x}+\left(\omega_{0}^{2}+h \cos \omega_{p} t\right) x+\alpha x^{3}+\eta x^{2} \dot{x}=0,
$$

where $x$ represents the displacement of the resonator. The normal frequency of the resonator is $\omega_{0} ; \alpha$ is the nonlinear spring constant, or Duffing parameter; $\gamma$ is the linear damping rate; and $\eta$ is the coefficient of nonlinear damping. The parameters $h$ and $\omega_{p}$ are the parametric driving amplitude and driving frequency, often called the pump frequency; these are easily varied in experimental implementations.

We concentrate on the so-called first instability tongue, operating near twice the resonance frequency $\omega_{p}=$ $2 \omega_{0}+\Omega$, with $h$ sufficiently large to overcome the damping [6]. If we fix all other parameters, the response amplitude $|a|$ of the resonator as a function of the relative frequency $\Omega$, follows the curve shown in Fig. 1(a). For $\alpha>0$, as in the case of doubly-clamped beams, the response amplitude of the driven resonator increases with increasing frequency, until it reaches a saddle-node bifurcation and drops abruptly to zero. The phase of the response, which is not plotted, is determined only to within a $\pi$ phase shift due to the fact that the drive completes two periods whenever the resonator completes only a single period at half the frequency. Chan et al. [11] studied noise-induced switching between these two oscillating states, differing by a $\pi$ phase shift, and Mahboob and Yamaguchi [12] proposed to employ them for memory storage.

Our coupled-nanoelectromechanical systems (NEMS) BTA consists of two resonators with slightly different resonance frequencies $\omega_{-}$and $\omega_{+}$, which are excited simultaneously using the same drive source. Given the $\pi$ phase freedom in the response of each resonator, when the resonators are uncoupled there is a $50 \%$ chance that they will respond in phase when both are excited, and a 50\% chance that they will respond with opposite phase. Consequently, in this case the summed response of the 

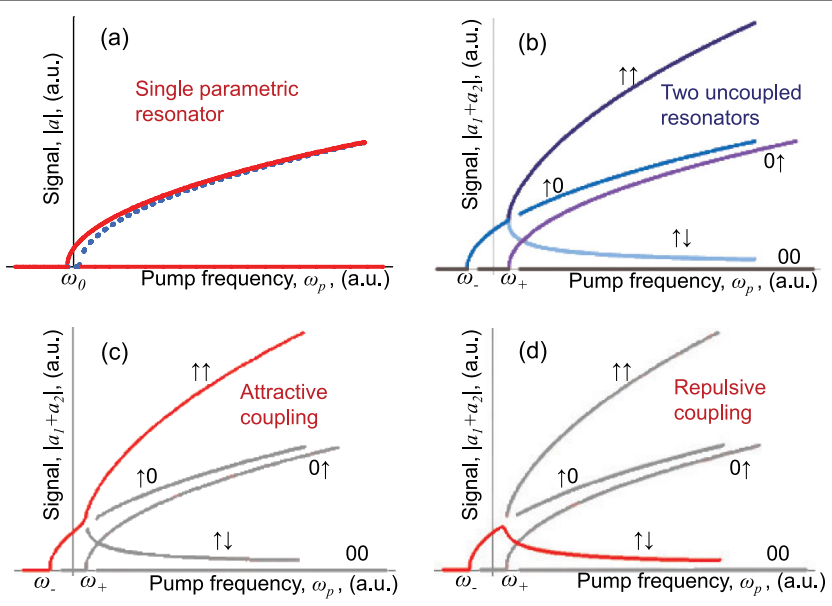

FIG. 1 (color online). (a) Response amplitude of a single parametric resonator given by (1). Stable solutions are in solid (red); unstable in dotted (blue). (b)-(d) Summed response of two resonators. Only the stable branches are shown, where $(00)$ is the zero state; in ( $\uparrow 0$ ) only the lower-frequency resonator is excited; in $(0 \uparrow)$ only the higher-frequency resonator is excited; in ( $\uparrow)$ both resonators are excited in phase, and in ( $\downarrow \downarrow)$ both resonators are excited in antiphase. (b) No coupling; (c) Attractive coupling; (d) Repulsive coupling. See supplementary information [13] for more details.

two resonators, $\left|a_{1}+a_{2}\right|$, forms a pitchfork bifurcation, as shown in Fig. 1(b), where the incoming branch-in which only the low-frequency resonator is excited-is connected to both the in-phase and the antiphase branches as the second resonator becomes excited. This behavior is confirmed experimentally in Fig. 2(a), as described below.

This situation changes when coupling is introduced between the resonators. Coupling alters the topology of the bifurcation diagram, causing a distortion of the perfect pitchfork. To illustrate this simply, we take the coupling to be linear, of the form $D\left(x_{1}-x_{2}\right)$ in Eq. (1) for the first resonator $x_{1}$, and with the interchange of indices in the corresponding equation for $x_{2}$. With attractive coupling $(D>0)$, as shown in Fig. 1(c), the incoming branch is connected only to the in-phase branch. With repulsive coupling $(D<0)$, as shown in Fig. 1(d), the opposite occurs, and the incoming branch is connected only to the antiphase branch. An upward quasistatic sweep of the pump frequency that starts to the left of the bifurcation will therefore easily distinguish between attractive and repulsive coupling. The summed response will follow the upper branch if the coupling is attractive-with the resonators oscillating in phase-and will follow the lower branch if the coupling is repulsive-with the resonators oscillating in antiphase. This dependence of the bifurcation topology on the effective coupling $D$ between resonators can be used to realize a novel method of signal amplification. To do so one employs $D$ as a control parameter, setting it proportional to a small input signal $V_{\text {in }}$ to be amplified.

We have realized a BTA using a pair of $6 \mu \mathrm{m} \times$ $500 \mathrm{~nm} \times 200 \mathrm{~nm}$ doubly clamped GaAs beams, separated by $400 \mathrm{~nm}$, as shown in the inset of Fig. 2(a) (see supple-
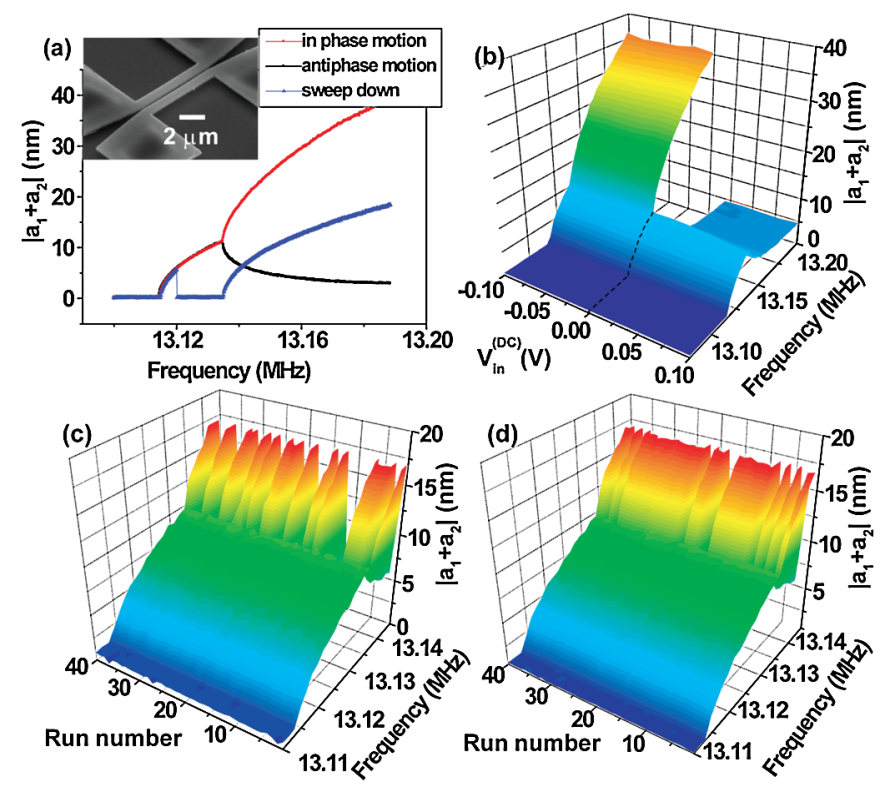

FIG. 2 (color online). (a) Experimental measurement of the parametric response of two uncoupled nanomechanical beams, confirming the pitchfork bifurcation [cf. Figure 1(b)]. (b) With a constant input signal $V_{\text {in }}^{(\mathrm{dc})}$ larger than the noise floor, the response to upward frequency sweeps deterministically follows the upper or lower curves depending on the sign of $V_{\mathrm{in}}^{(\mathrm{dc})}$. (c) Repeated upward frequency scans with no coupling, $V_{\text {in }}^{(\mathrm{dc})}=$ 0 , show that each of the two possible branches are followed with equal probability. (d) Repeated scans with $V_{\text {in }}^{(\mathrm{dc})}=-3 \mathrm{mV}$, show that a majority of the scans follow the upper in-phase response curve, yet because the noise amplitude is greater than $3 \mathrm{mV}$, some scans still follow the lower antiphase response curve.

mentary information [13] for more details). The fundamental out-of-plane resonance frequencies of the devices are around $13.1 \mathrm{MHz}$, differing by about $30 \mathrm{kHz}$ as a result of small fabrication variations. Resonance quality factors in vacuum at room temperature are approximately 1700 . The GaAs beams consist of a layered structure [9] that develops longitudinal strain when a voltage $V_{\text {app }}$ is applied between their top and bottom surfaces. When clamped at both ends, this strain is converted into stress, which either stiffens or softens the beams thus tuning their natural resonance frequencies. Any applied voltage $V_{\text {app }}$ also induces electric dipoles within the layers of the beams [14], which causes the beams to repel each other, thus making the coupling $D$ more negative. In the absence of an applied voltage there is residual attraction between the beams due to elastic coupling through their shared elastic support. A positive dc offset voltage $V_{\text {offset }} \sim 1.1 \mathrm{~V}$ precisely compensates this elastic attraction for these devices and nulls the intrinsic coupling between the beams. Thus, we set the effective coupling between the beams $V_{\text {in }}=V_{\text {app }}-V_{\text {offset }}$, which is proportional to $-D$, as our input signal.

We measure the summed out-of-plane displacement amplitude of the beams $\left|a_{1}+a_{2}\right|$ by optical interferometry using a laser beam that illuminates both resonators. The interference signal is strongest when the beams oscillate in 
phase and weakest when they oscillate in antiphase. We subsequently translate the interferometer output signal into motional amplitude, calibrated in nanometers, using the known value of the critical amplitude at the onset of nonlinear response for driven Duffing resonators [6,15]. Figure 2(a) shows the summed response of the two beams measured for zero effective coupling, $V_{\text {in }}=0$. These data confirm the existence of the pitchfork bifurcation, predicted in Fig. 1(b). Figure 2(b) shows a sequence of upward frequency sweeps for finite dc input signals that are stronger than the background noise, confirming the predictions of Figs. 1(c) and 1(d), that the device acts as a very sensitive discriminator between positive and negative input signals. Its resolution is limited only by the amplitude of background fluctuations, which comprise noise accompanying the input signal itself, thermomechanical motion of the beams, and other sources as described in [13]. For signals that are weaker than the noise, there is a finite probability of following the "wrong" branch, and fluctuations ultimately determine which branch is followed. At precisely $V_{\text {in }}=0$, the probabilities to follow the two branches are equal, as shown in Fig. 2(c). This balance tips in favor of the lower branch for $V_{\text {in }}^{(\mathrm{dc})}>0$, and in favor of the upper branch for $V_{\mathrm{in}}^{(\mathrm{dc})}<0$, as evident in Fig. 2(d) for $V_{\text {in }}^{(\mathrm{dc})}=-3 \mathrm{mV}$. In fact, as explained in [13] and demonstrated below, for small input-signal-to-noise ratio, the probability of following the "correct" branch is linear in the signal, and the BTA indeed acts as a linear amplifier.

Our measurement setup to realize a BTA is shown in Fig. 3(a)[13]. Three waveforms with a decreasing hierarchy of frequencies are applied to the device: (a) A fast rf drive at frequency $\omega_{p}$, which parametrically pumps the beams; (b) a triangular sweep waveform, at an intermediate frequency $\omega_{\mathrm{sw}}$, that is used to frequency modulate the rf pump voltage and sweep it through the bifurcation point; and (c) a slower input signal $V_{\text {in }}(t)$ to be amplified. The sweep waveform provides fast and consecutive linearlyramped frequency sweeps that start below the bifurcation point and end above it. At the beginning of each upward sweep the BTA is thereby reset, enabling it to sample anew the sign of the input signal $V_{\text {in }}(t)$.

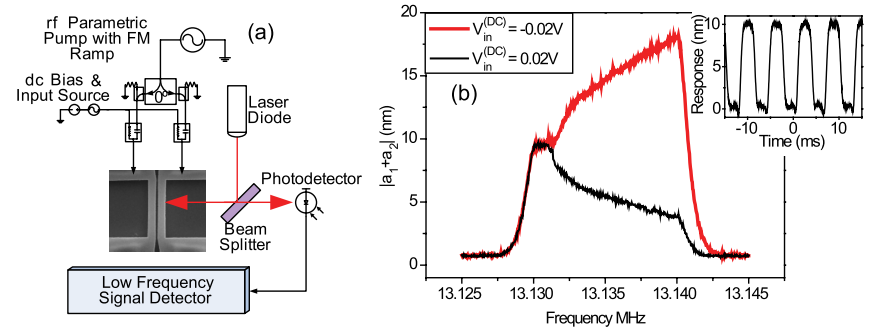

FIG. 3 (color online). (a) Schematics of the experimental setup. (b) The output response measured by a spectrum analyzer. The upper (red) spectral response curve is obtained for a negative input signal, and the lower (black) spectral response curve for a positive input signal. Inset: Demodulated output signal for a square-wave input signal.
There are fundamental constraints on the sweep rate $2 \omega_{\text {in }}^{(\max )}<\omega_{\text {sw }} \ll \gamma$ set by the Nyquist criterion and requiring $\omega_{\mathrm{sw}}$ to be slower than the resonators' relaxation rate $\gamma$. The latter ensures that the system adiabatically follows the topology of the bifurcation diagram, which is essential for proper BTA operation. For the experimental resonance frequency of $13.1 \mathrm{MHz}$ and quality factors of $\sim 1700$, the resonators' response times are of order $0.1 \mathrm{~ms}$. In our measurements, we find that the output signal diminishes for sweep rates exceeding $\sim 2 \mathrm{kHz}$, corresponding to relaxation times around $0.5 \mathrm{~ms}$, in agreement with this estimate. We anticipate that significant increases in signal bandwidth up to $\mathrm{MHz}$ scales should be readily attainable using previously demonstrated NEMS resonators at microwave frequencies.

Figure 3(b) shows the measured spectral response of the BTA for a positive input signal (lower curve) and for a negative input signal (upper curve). The response of the photodetector of the interferometer is an rf output voltage that oscillates at the resonant frequency of the beams, with amplitude proportional to the summed displacement of the beams. We define the BTA output as the signal obtained by homodyne conversion of the photodetector signal using a local oscillator synchronized to $\omega_{p}$, but at half the pump frequency, $\omega_{p} / 2$. This yields a demodulated, amplified, BTA-sampled replica of the input signal. The inset of Fig. 3(b) displays the demodulated output for a squarewave input signal. The measured BTA output is the rms summed displacements of the two resonators, averaged over many sweeps. To within a constant shift, this is proportional to the probability $P_{\uparrow}$ of following the upper branch minus the probability $P_{\downarrow}$ of following the lower branch. This difference in probabilities is shown [13] to follow an error function.

We characterize our BTA by amplifying a simple input signal $V_{\text {in }}(t)=V_{\text {in }}^{(\mathrm{dc})}+V_{\text {in }}^{(\mathrm{ac})}(t)$, where $V_{\text {in }}^{(\mathrm{ac})}(t)$ is a sinusoidal or a square wave with amplitude $V_{\mathrm{in}}^{(\mathrm{ac})}$ and frequency $\omega_{\text {in }} / 2 \pi=167 \mathrm{~Hz}$, offset from zero by a finite dc shift $V_{\text {in }}^{(\mathrm{dc})}$. A sweep rate of $\omega_{\text {sw }} / 2 \pi=557 \mathrm{~Hz}$ is employed to sample the output signal, and a lock-in amplifier is employed for taking the BTA output and extracting the amplitude of its modulation at the frequency of $V_{\text {in }}^{(\mathrm{ac})}(t)$. Figure 4(a) displays a family of curves showing the modulation amplitude of the BTA output, measured as a function of $V_{\text {in }}^{(\mathrm{dc})}$, for a set of fixed input amplitudes $V_{\text {in }}^{(\mathrm{ac})}$. For large $\left|V_{\text {in }}^{(\mathrm{dc})}\right|$ relative to $V_{\text {in }}^{(\mathrm{ac})}$ the BTA output is not at all modulated, giving rise to the widths of the observed peaks in Fig. 4(a), while the sharpness of the rise and fall of the curves depends on the noise amplitude. The maximum peak height, obtained for $V_{\text {in }}^{(\mathrm{dc})}=0$, which is proportional to $P_{\uparrow}-P_{\downarrow}$, is plotted in Fig. 4(b) as a function of the input amplitude $V_{\mathrm{in}}^{(\text {ac) }}$, showing the expected error function dependence. For input amplitudes exceeding the noise, $V_{\text {in }}^{(\text {ac })}>20 \mathrm{mV} \mathrm{rms}$, the maximum peak heights saturate 


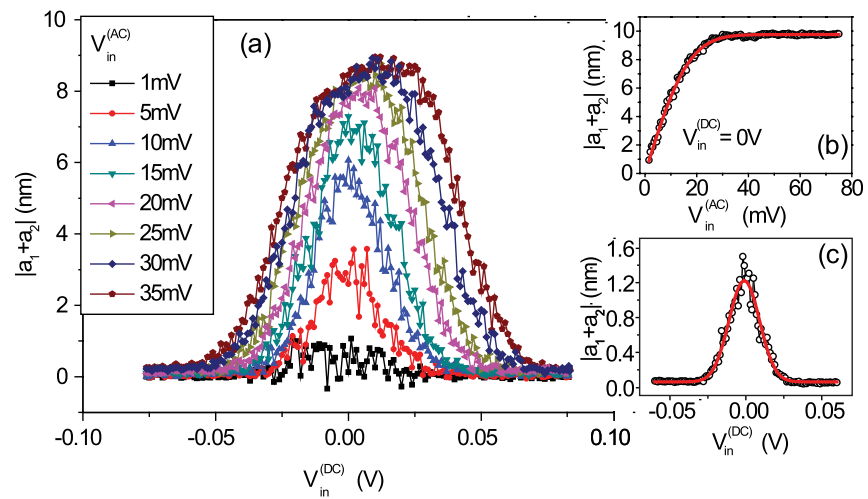

FIG. 4 (color online). (a) BTA output for different values of the signal amplitude $V_{\text {in }}^{(\text {ac) }}$, as a function of its offset $V_{\text {in }}^{(\mathrm{dc})}$ from zero. (b) BTA output as a function of the input amplitude $V_{\text {in }}^{(\text {ac) }}$, for zero offset voltage. The response closely matches the theoretically predicted error function [13], shown as a solid (red) line. (c) The smallest measured signal in (a), with $V_{\text {in }}^{(\mathrm{ac})}=1 \mathrm{mV}$ corresponding to about $70 e$ applied to each resonator's conducting layer, averaged for a longer time corresponding to an integration bandwidth of $0.056 \mathrm{~Hz}$.

because the BTA deterministically switches between the two branches in every period of the input signal. For small input amplitudes the average output signal is linear, with a slope that depends on noise. An increase in the noise amplitude will decrease the slope, thereby delaying the saturation of the BTA response and extending the dynamic range but at the cost of suppressed gain.

The utility of the BTA clearly depends on its particular implementation. Here, with our coupled-NEMS BTA as an example, we can estimate its sensitivity as a charge detector. We easily resolve an input signal $V_{\text {in }}^{(\text {ac })}=1 \mathrm{mV}$ corresponding to $\sim 70 e$ of charge on the resonators' electrodes $\left(e=1.6 \times 10^{-19} \mathrm{C}\right)$. Figure $4(\mathrm{c})$ shows that a $0.056 \mathrm{~Hz}$ integration bandwidth yields a signal-to-noise ratio of $\sim 100$. A detailed analysis of the noise [13], shows that this demonstrates a charge noise spectral density for this BTA of $\sqrt{S_{q}} \sim 30 e / \sqrt{\mathrm{Hz}}$. The supplementary information [13] also contains analyses of the principal noise sources limiting coupled-NEMS BTA performance. These indicate that very significant enhancement, ultimately limited by fundamental noise sources, should be attainable [16]. In this limit, we estimate that charge sensitivity will improve to a level of order $\sim 0.5 e / \sqrt{\mathrm{Hz}}$ for our current device at room temperature, and $\sim 10^{-5} e / \sqrt{\mathrm{Hz}}$ for an optimized device with $\mathrm{GHz}$ frequency and reduced capacitance, operating at cryogenic temperatures.

The first implementation of BTA principles, described here, is based on coupled-NEMS resonators. However, the simple and generic principles underlying the BTA suggest its applicability to a wide variety of physical systems, such as laser cavities, superconducting resonators, coupled Josephson junctions, and possibly even to oscillating chemical and biological systems.
This research is partially supported by the National Science Foundation under Grant No. DMR-0314069, and the US-Israel Binational Science Foundation (BSF) under Grant No. 2004339. We thank Philip Feng and Inna Kozinsky for useful discussions, and Iwijn De Vlaminck and Gustaaf Borghs from IMEC (Leuven, Belgium) for providing us with the GaAs material.

\section{*ronlif@tau.ac.il \\ †roukes@caltech.edu}

[1] L. A. Wu, H. J. Kimble, J. L. Hall, and H. Wu, Phys. Rev. Lett. 57, 2520 (1986); D. Rugar and P. Grütter, ibid. 67, 699 (1991); D. Carr, S. Evoy, L. Sekaric, H. Craighead, and J. Parpia, Appl. Phys. Lett. 77, 1545 (2000); G. Breitenbach, S. Schiller, and J. Mlynek, Nature (London) 387, 471 (1997).

[2] P. G. Kwiat, K. Mattle, H. Weinfurter, A. Zeilinger, A. V. Sergienko, and Y. Shih, Phys. Rev. Lett. 75, 4337 (1995).

[3] W. M. Dougherty, K. J. Bruland, J. L. Garbini, and J. A. Sidles, Meas. Sci. Technol. 7, 1733 (1996).

[4] K. L. Turner, S. A. Miller, P.G. Hartwell, N.C. MacDonald, S. H. Strogatz, and S. G. Adams, Nature (London) 396, 149 (1998).

[5] E. Buks and M.L. Roukes, J. Microelectromech. Syst. 11, 802 (2002).

[6] R. Lifshitz and M.C. Cross, in Review of Nonlinear Dynamics and Complexity, edited by H.G. Schuster (Wiley, Meinheim, 2008), Vol. 1, pp. 1-52.

[7] R. Lifshitz and M.C. Cross, Phys. Rev. B 67, 134302 (2003); Y. Bromberg, M. C. Cross, and R. Lifshitz, Phys. Rev. E 73, 016214 (2006); E. Kenig, R. Lifshitz, and M. C. Cross, ibid. 79, 026203 (2009); E. Kenig, B. A. Malomed, M.C. Cross, and R. Lifshitz, ibid. 80, 046202 (2009); R. B. Karabalin, M.C. Cross, and M.L. Roukes, Phys. Rev. B 79, 165309 (2009).

[8] K. Wiesenfeld and B. McNamara, Phys. Rev. A 33, 629 (1986); I. Siddiqi, R. Vijay, F. Pierre, C. M. Wilson, M. Metcalfe, C. Rigetti, L. Frunzio, and M. H. Devoret, Phys. Rev. Lett. 93, 207002 (2004); R. Almog, S. Zaitsev, O. Shtempluck, and E. Buks, Appl. Phys. Lett. 88, 213509 (2006); E. Buks and B. Yurke, Phys. Rev. E 74, 046619 (2006).

[9] S.C. Masmanidis, R.B. Karabalin, I. De Vlaminck, G. Borghs, M. R. Freeman, and M. L. Roukes, Science 317, 780 (2007).

[10] D. W. Carr and H. G. Craighead, J. Vac. Sci. Technol. 15, 2760 (1997).

[11] H. B. Chan, M. I. Dykman, and C. Stambaugh, Phys. Rev. E 78, 051109 (2008).

[12] I. Mahboob and H. Yamaguchi, Nature Nanotech. 3, 275 (2008).

[13] See supplementary material at http://link.aps.org/ supplemental/10.1103/PhysRevLett.106.094102.

[14] H.X. Tang, X. M. H. Huang, M. L. Roukes, M. Bichler, and W. Wegscheider, Appl. Phys. Lett. 81, 3879 (2002).

[15] H.W.C. Postma, I. Kozinsky, A. Husain, and M.L. Roukes, Appl. Phys. Lett. 86, 223105 (2005).

[16] A. N. Cleland and M. L. Roukes, J. Appl. Phys. 92, 2758 (2002); A. N. Cleland, New J. Phys. 7, 235 (2005). 